\title{
A UNIFIED TREATMENT OF DIVIDEND PAYMENT PROBLEMS UNDER FIXED COST AND IMPLEMENTATION DELAYS
}

\author{
ERHAN BAYRAKTAR AND MASAHIKO EGAMI
}

\begin{abstract}
In this paper we solve the dividend optimization problem for a corporation or a financial institution when the managers of the corporation are facing (regulatory) implementation delays. We consider several cash reservoir models for the firm including two mean-reverting processes, Ornstein-Uhlenbeck and square-root processes. Since the cashflow structure of different companies have different qualitative behaviors it makes sense to use different diffusions to model them. We provide a unified mathematical framework to analyze all these models and find the optimal barrier strategies. Our solution depends on a new characterization of the value function for one-dimensional diffusions and provide easily implementable algorithms to find the optimal control and the value function.
\end{abstract}

\section{INTRODUCTION}

In this paper, we solve the dividend optimization problem for a corporation or a financial institution. The corporation controls the timing and the amount of dividends and the objective of the corporation is to maximize the total discounted dividends paid out to shareholders until the time of bankruptcy given that the dividend payments are subject to regulatory delay. The payment of a dividend is not automatic and payments can be made only after a certain amount of time elapses. The amount and the timing of payment is decided by the company managers but these are subject to the approval of the company's owners (shareholders) and maybe also of debt holders and therefore it takes some time before the dividends are paid. Recently, there have been other papers on optimally controlling a state variable subject to implementation delays in different modeling contexts, see e.g. [2], [3], [4], [16], [21] and [25]. Our methodolgy of solving this problem is in the spirit of [4] and differs from the other papers cited above as will be made clear below.

We model the problem of the corporation as an impulse control problem and assume that when dividend is paid out, the firm has to pay a fixed cost representing the resources it has to devote to the distribution of dividends. This amount is independent of the size of the dividend payment. Other papers modeling the dividend payment problem as an impulse control problem are [7], [13] and [23]. There are several other papers which model the dividend payment problem as a singular stochastic control problem by assuming that there is no fixed cost at the time of dividend payment; see e.g. [10], [11], [12], [13] and $[26]$.

2000 Mathematics Subject Classification. Primary: 93E20, Secondary: 60J60.

Key words and phrases. Impulse Control, Implementation Delay, Dividend Payments, Brownian motion, OrnsteinUhlenbeck Process, Square-root Process, Itô Diffusions.

E. Bayraktar is supported in part by the National Science Foundation. 
Applying an appropriate transformation to the value of a particular control, we transform the problem into a non-linear programming problem. Using the new characterization of the value function we give an easy to implement algorithm to determine the optimal control and the value function. A secondary result of our paper are the sufficient conditions under which the smooth fit holds (see Remark 4.1 and Proposition 4.1). In contrast, in the literature impulse control problems are solved first finding a classical solution to a system of quasi variational inequalities. The optimal thresholds are determined using the so-called "smooth fit principle" (by hypothesizing that the smooth fit holds). (Once a classical solution to this system is determined it can be shown to be equal to the value function by the so called verification lemma.) See e.g. Bensoussan and Lions [5] and Øksendal and Sulem [20].

In this paper, the time horizon is the time of ruin, and this makes the analysis more difficult from that of [4], which only considers infinite horizon problems. Since cashflow of different companies have different qualitative behavior, a manager needs a portfolio of tractable models to choose from. Here we consider four models for the aggregate income/cash reservoir of the firm: i) Brownian motion with drift, ii) Ornstein-Uhlenbeck, iii) Square-root process, iv) Geometric Brownian motion. Most of the papers related to stochastic impulse control, in order to obtain analytical solutions, assume that the uncontrolled process is a Brownian motion with drift. In addition to using Brownian motion to model the cash reservoir, we also propose two mean reverting processes as possible modeling alternatives which is suggested by the Cash Flow Hypothesis in Jensen [14]; see [12] for further motivation. On the other hand, geometric Brownian motion is used to model the firm value in the structural models in credit risk modeling. Our solution for the geometric Brownian motion model can also be interpreted as the optimal dividend distribution to the stockholders of a given company since geometric Brownian motion is frequently used to model the value of a company (for e.g. in the structural credit risk models) [18]; see [10] for further motivation. As far as we know, our paper is the first one that explicitly handles the dividend payment problem for for the square root process (with or without delays).

The rest of the paper is organized as follows: In Section 2, we present the models for the cash reservoir and state the dividend payment problem. In Section 3, we provide a characterization of the value function for a given threshold strategy. In Section 4, we provide an easily implementable algorithm to find the optimal threshold strategy. We also provide theoretical justification for our algorithm in this section (see e.g. Proposition 4.1). We then check that the models satisfy the sufficient assumptions of optimality in Section 4.3. Finally, in Section 5 we present some numerical examples.

\section{Statement of The Problem}

Let $(\Omega, \mathcal{F}, \mathbb{P})$ be a complete probability space with a standard Brownian motion $W=\left\{W_{t} ; t \geq 0\right\}$. We model the aggregate income process $X^{0}$ as either the Brownian motion

$$
d X_{t}^{0}=\mu d t+\sigma d W_{t}, \quad X_{0}^{0}=x>0,
$$

for some constants $\mu, \sigma>0$; or the Ornstein Uhlenbeck process

$$
d X_{t}^{0}=-\rho X_{t}^{0} d t+d W_{t}, \quad X_{0}^{0}=x>0,
$$


for some constant $\rho>0$, or the square root process

$$
d X_{t}^{0}=\left(1-2 \rho X_{t}^{0}\right) d t+2 \sqrt{X_{t}^{0}} d W_{t}, \quad X_{0}^{0}=x>0 .
$$

Note that if the initial condition of (2.3) is properly chosen, then the solution of it is the square of the solution of (2.2). We will also consider the case when the aggregate income process follows the geometric Brownian motion

$$
d X_{t}^{0}=\mu X_{t}^{0} d t+\sigma X_{t}^{0} d W_{t}, \quad X_{0}^{0}=x>0 .
$$

The firm will pay dividends to its shareholders out of the aggregate income process $X^{0}$ and the net holdings of the firm, i.e. the net income process will be denoted by $X$. We assume that the company pays out dividends to its shareholders in order to maximize the expected value of discounted dividends paid out until the time of ruin. There will be a fixed amount of transaction cost for making a dividend payment. In this framework a dividend payment scheme that a firm follows can be represented by a doubly stochastic sequence

$$
\nu=\left(T_{1}, T_{2}, \ldots . T_{i} \ldots ; \xi_{1}, \xi_{2}, \ldots \xi_{i} \ldots\right),
$$

where $0 \leq T_{1}<T_{2}<\ldots$ is an increasing sequence of $\mathbb{F}$-stopping times such that $T_{i+1}-T_{i} \geq \Delta$, and $\xi_{1}, \xi_{2} \ldots$ are $\mathcal{F}_{\left(T_{i}+\Delta\right)-}$ measurable random variables representing the dividend amount paid out. The firm decides to make dividend payments at (random) time $T_{i}$, but it can not act until time $T_{i}+\Delta$ (where $\Delta \geq 0$ is a constant). It decides on the magnitude of the dividend amount at $T_{i}+\Delta$ depending on the level of its revenues. We will in particular consider benchmark strategies. These strategies are determined by specifying two numbers $0 \leq a<b$ as follows: At the time the aggregate profit (or the firm value) hits a large enough level $b$, the shareholders ask the firm to commit to making dividend payments and reduce the level of net profits (or the firm value) to $a$. We denote by $\mathcal{V}$ the set of strategies that fit into this description. We will refer to them as the admissible strategies.

The net income process follows (until after the first dividend payment)

$$
\left\{\begin{array}{l}
d X_{t}=\mu\left(X_{t}\right) d t+\sigma\left(X_{t}\right) d W_{t}, \quad 0 \leq t<T_{1}+\Delta, \\
X_{T_{1}+\Delta}=X_{\left(T_{1}+\Delta\right)-}-\xi_{1},
\end{array}\right.
$$

for appropriate functions $\mu$ and $\sigma$ depending on which case we are inspecting. For the first three cases we assume that 0 is the absorbing state and define $\tau_{0}$ (the time of ruin) as :

$$
\tau_{0} \triangleq \inf \left\{t \geq 0: X_{t} \leq 0\right\} .
$$

When the aggregate income process follows the geometric Brownian motion, the time of ruin is defined as

$$
\tau_{d} \triangleq \inf \left\{t \geq 0: X_{t} \leq d\right\},
$$

for some fixed $d>0$. The purpose of the firm is to maximize expected value of the discounted dividend payments until the time of ruin, i.e.,

$$
J^{\nu}(x) \triangleq \mathbb{E}^{x}\left[\sum_{T_{i}+\Delta<\tau_{0}} e^{-\alpha\left(T_{i}+\Delta\right)} K\left(X_{\left(T_{i}+\Delta\right)-}, X_{T_{i}+\Delta}\right)\right],
$$


over all the admissible strategies. We will assume that

$$
K\left(X_{\left(T_{1}+\Delta\right)-}, X_{T_{1}+\Delta}\right)=X_{\left(T_{1}+\Delta\right)-}-X_{T_{1}+\Delta}-\lambda
$$

where $\lambda>0$ is a fee associated with a transaction. We could also consider

$$
K\left(X_{\left(T_{1}+\Delta\right)-}, X_{T_{1}+\Delta}\right)=k \cdot\left(X_{\left(T_{1}+\Delta\right)-}-X_{T_{1}+\Delta}\right)-\lambda
$$

for $k \in(0,1)$, in which $1-k$ can be considered as the tax rate. This does not affect the analysis and therefore we will focus on the case when $k=1$.

Let us denote the value function of this problem by

$$
v(x) \triangleq \sup _{\nu \in \mathcal{V}} J^{\nu}(x)=J^{\nu^{*}}(x)
$$

When $X^{0}$ is the Ornstein Uhlenbeck process, in addition to considering the performance function in (2.7) we will also consider the following performance

$$
J^{\nu}(x) \triangleq \mathbb{E}^{x}\left[\sum_{T_{i}+\Delta<\tau_{0}} e^{-\alpha\left(T_{i}+\Delta\right)} K\left(X_{\left(T_{i}+\Delta\right)-}, X_{T_{i}+\Delta}\right)-P e^{-\alpha \tau_{0}}\right]
$$

for some constant $P>0$. The rationale for considering this penalty function is to penalize declaring banktruptcy. As we shall see if the purpose is to maximize the performance function in (2.7), when $X^{0}$ follows an OU process, it is optimal to declare bankruptcy when the aggregate income process reaches a certain level. Therefore the OU process might be used to model the income process of firms in distress. This might give an idea to the creditors of how this type of a firm might behave. The extra cost in (2.9) will, on the other hand, deter the firms from declaring bankruptcy.

\section{Characterization of the Value Function}

In this section, we will show that when we apply a suitable transformation (see 3.6) to the value function corresponding to a particular threshold strategy (that is identified by a pair $(a, b)$ ), the transformed value function is linear on $(F(0), F(b))$. This characterization will become important in determining the optimal 
threshold strategy in the next section. Equation (2.7) can be developed as

$$
\begin{aligned}
& J^{\nu}(x)=\mathbb{E}^{x}\left[\sum_{T_{i}+\Delta<\tau_{0}} e^{-\alpha\left(T_{i}+\Delta\right)} K\left(X_{\left(T_{i}+\Delta\right)-}, X_{T_{i}+\Delta}\right)\right] \\
& =\mathbb{E}^{x}\left[1 _ { \{ T _ { 1 } + \Delta < \tau _ { 0 } \} } \left\{e^{-\alpha\left(T_{1}+\Delta\right)} K\left(X_{\left(T_{1}+\Delta\right)-}, X_{T_{1}+\Delta}\right)\right.\right. \\
& \left.\left.+e^{-\alpha\left(T_{1}+\Delta\right)} \sum_{i \geq 1: T_{i}+\Delta<\tau_{0},} e^{-\alpha\left(\left(T_{i+1}+\Delta\right)-\left(T_{1}+\Delta\right)\right)} K\left(X_{\left(T_{i+1}+\Delta\right)-}, X_{T_{i+1}+\Delta}\right)\right\}\right]
\end{aligned}
$$

where we used $T_{i+1}+\Delta=\left(T_{1}+\Delta\right)+\left(T_{i}+\Delta\right) \circ \theta\left(T_{1}+\Delta\right)$ with the shift operator $\theta(\cdot)$ in the second equality.

Since $T_{1}=\tau_{b}$ with $\tau_{b}=\inf \left\{t \geq 0: X_{t}^{0} \geq b\right\}$ and the post intervention point by

$$
X_{T_{1}+\Delta}=X_{\tau_{b}+\Delta}=X_{\left(\tau_{b}+\Delta\right)-}-\xi_{1} \triangleq a .
$$

It follows that

$$
\begin{aligned}
J^{\nu}(x) & =\mathbb{E}^{x}\left[1_{\left\{\tau_{b}+\Delta<\tau_{0}\right\}} e^{-\alpha\left(\tau_{b}+\Delta\right)}\left\{K\left(X_{\left(\tau_{b}+\Delta\right)-}, a\right)+J^{\nu}(a)\right\}\right] \\
& =\mathbb{E}^{x}\left[\mathbb{E}^{x}\left[1_{\left\{\tau_{b}+\Delta<\tau_{0}\right\}} e^{-\alpha\left(\tau_{b}+\Delta\right)}\left\{K\left(X_{\left(\tau_{b}+\Delta\right)-}, a\right)+J^{\nu}(a)\right\} \mid \mathcal{F}_{\tau_{b}}\right]\right] \\
& =\mathbb{E}^{x}\left[1_{\left\{\tau_{b}<\tau_{0}\right\}} e^{-\alpha \tau_{b}} \mathbb{E}^{X_{\tau_{b}}}\left[1_{\left\{\Delta<\tau_{0}\right\}} e^{-\alpha \Delta}\left\{K\left(X_{\Delta-}, a\right)+J^{\nu}(a)\right\}\right]\right] .
\end{aligned}
$$

Evaluating $J^{\nu}$ at $x=b$, we obtain

$$
J^{\nu}(b)=\mathbb{E}^{b}\left[1_{\left\{\Delta<\tau_{0}\right\}} e^{-\alpha \Delta}\left(K\left(X_{\Delta_{-}}, a\right)+J^{\nu}(a)\right)\right],
$$

and evaluating $J^{\nu}$ at $x=0$ we get $J^{\nu}(0)=0$.

Now, we can write $(3.2)$ as

$$
J^{\nu}(x)= \begin{cases}\mathbb{E}^{x}\left[1_{\left\{\tau_{b}<\tau_{0}\right\}} e^{-\alpha \tau_{b}} J^{\nu}(b)+1_{\left\{\tau_{b}>\tau_{0}\right\}} e^{-\alpha \tau_{0}} J^{\nu}(0)\right] & 0 \leq x \leq b \\ \mathbb{E}^{x}\left[1_{\left\{\Delta<\tau_{0}\right\}} e^{-\alpha \Delta}\left(K\left(X_{\Delta}, a\right)+J^{\nu}(a)\right)\right] & x>b .\end{cases}
$$

Similarly, if the performance function to minimize is the one defined in (2.9), then we have

$$
J^{\nu}(x)= \begin{cases}\mathbb{E}^{x}\left[1_{\left\{\tau_{b}<\tau_{0}\right\}} e^{-\alpha \tau_{b}} J^{\nu}(b)+1_{\left\{\tau_{b}>\tau_{0}\right\}} e^{-\alpha \tau_{0}} J^{\nu}(0)\right], & 0 \leq x \leq b, \\ \mathbb{E}^{x}\left[1_{\left\{\Delta<\tau_{0}\right\}} e^{-\alpha \Delta}\left(K\left(X_{\Delta}, a\right)+J^{\nu}(a)\right)-P 1_{\left\{\Delta>\tau_{0}\right\}} e^{-\alpha \tau_{0}}\right] & x>b .\end{cases}
$$


In this case $J^{\nu}(0)=-P$ and

$$
J^{\nu}(b)=\mathbb{E}^{b}\left[1_{\left\{\Delta<\tau_{0}\right\}} e^{-\alpha \Delta}\left(K\left(X_{\Delta}, a\right)+J^{\nu}(a)\right)-P 1_{\left\{\Delta>\tau_{0}\right\}} e^{-\alpha \tau_{0}}\right] .
$$

We will denote the infinitesimal generator of the process $X^{0}$ by $\mathcal{A}$. Let us denote the increasing and decreasing solutions of the second-order ordinary differential equation $(\mathcal{A}-\alpha) u=0$ by $\psi(\cdot)$ and $\varphi(\cdot)$ respectively (these are uniquely determined up to a multiplication). We can write

$$
\mathbb{E}^{x}\left[e^{-\alpha \tau_{r}} 1_{\left\{\tau_{r}<\tau_{l}\right\}}\right]=\frac{\psi(l) \varphi(x)-\psi(x) \varphi(l)}{\psi(l) \varphi(r)-\psi(r) \varphi(l)}, \mathbb{E}^{x}\left[e^{-\alpha \tau_{l}} 1_{\left\{\tau_{l}<\tau_{r}\right\}}\right]=\frac{\psi(x) \varphi(r)-\psi(r) \varphi(x)}{\psi(l) \varphi(r)-\psi(r) \varphi(l)},
$$

for $x \in[l, r]$ where $\tau_{l} \triangleq \inf \left\{t>0 ; X_{t}^{0}=l\right\}$ and $\tau_{r} \triangleq \inf \left\{t>0 ; X_{t}^{0}=r\right\}$ (see e.g. Dayanik and Karatzas [9]). Let us introduce the increasing function

$$
F(x) \triangleq \frac{\psi(x)}{\varphi(x)}
$$

By defining

$$
W \triangleq\left(J^{\nu} / \varphi\right) \circ F^{-1}
$$

on $x \in[0, b]$, using (3.5), equation (3.3) on $0 \leq x \leq b$ becomes

$$
W(F(x))=W(F(b)) \frac{F(x)-F(0)}{F(b)-F(0)}+W(F(0)) \frac{F(b)-F(x)}{F(b)-F(0)} \quad 0 \leq x \leq b
$$

which shows that the value function is linear in the transformed space. Next, we will compute

$$
B \triangleq \mathbb{E}^{x}\left[1_{\left\{\Delta<\tau_{0}\right\}} e^{-\alpha \Delta}\left(K\left(X_{\Delta}, a\right)+J^{\nu}(a)\right)\right]
$$

in (3.3) for all the different models of aggregate income process. (In the case of geometric Brownian motion we will replace $\tau_{0}$ by $\tau_{d}$ in (3.8). Moreover the function $J^{\nu}(x)$ for this case is given by replacing 0's with d's in (3.4).)

\subsection{Computation of $B$ in (3.8).}

3.1.1. Ornstein-Uhlenbeck Process. Let's first consider the case when $X^{0}$ is the Ornstein-Uhlenbeck process given by (2.2). Recall that $X_{t}^{0}$ can be written as (can be derived using Theorem 4.6 of Karatzas and Shereve [15])

$$
\begin{gathered}
X_{t}^{0}=x e^{-\rho t}+B_{Q(t)}, \quad \text { where } \quad Q(t)=\frac{1-e^{-2 \rho t}}{2 \rho}, \quad \text { or } \\
e^{\rho t} X_{t}^{0}=x+\tilde{B}_{\tilde{Q}(t)}, \quad \text { where } \quad \tilde{Q}(t)=\frac{e^{2 \rho t}-1}{2 \rho},
\end{gathered}
$$

and $B$ and $\tilde{B}$ are Brownian motions. This implies that the distribution of $X_{t}^{0}$ is $\operatorname{Gsn}\left(x e^{-\alpha t}, Q(t)\right)$. (We use $\operatorname{Gsn}(a, b)$ to denote a Gaussian random variable with mean $a$ and variance $b$.) As a result of the 
representation in (3.10)

$$
\begin{aligned}
\mathbb{P}^{x}\left(\tau_{0}>\Delta\right) & =\mathbb{P}^{x}\left(\tau_{0}^{\tilde{B}}>\tilde{Q}(\Delta)\right)=1-\frac{2}{\sqrt{2 \pi}} \int_{x / \sqrt{\tilde{Q}(\Delta)}}^{\infty} e^{-u^{2} / 2} d u \\
& =2 N\left(\frac{x}{\sqrt{\tilde{Q}(\Delta)}}\right)-1=2 N\left(\frac{x e^{-\rho \Delta}}{\sqrt{Q(\Delta)}}\right)-1,
\end{aligned}
$$

where $\tau_{0}^{\tilde{B}}$ is the first time the Brownian motion $x+\tilde{B}$ hits zero. Here, we used the distribution of the hitting times of Brownian motion (see page 96 of Karatzas and Shreve). We also used the notation that $N(x)=\int_{-\infty}^{x} 1 /(\sqrt{2 \pi}) e^{-u^{2} / 2} d u$.

Let us try to identify the density function of $Y_{\Delta} \triangleq X_{\Delta}^{0} 1_{\left\{\tau_{0}>\Delta\right\}}$. To this end we first compute

$$
\begin{aligned}
\mathbb{P}^{x}\left\{X_{\Delta}^{0} \geq y, \tau_{0}>\Delta\right\} & =\mathbb{P}^{x}\left\{X_{\Delta}^{0} \geq y\right)-\mathbb{P}^{x}\left(X_{\Delta}^{0} \geq y, \tau_{0} \leq \Delta\right\} \\
& =\mathbb{P}^{x}\left\{X_{\Delta}^{0} \geq y\right\}-\mathbb{P}^{x}\left\{X_{\Delta}^{0} \leq-y, \tau_{0} \leq \Delta\right\} \\
& =\mathbb{P}^{x}\left\{X_{\Delta}^{0} \geq y\right\}-\mathbb{P}^{x}\left\{X_{\Delta}^{0} \leq-y\right\} \\
& =\frac{1}{\sqrt{2 \pi Q(\Delta)}}\left(\int_{y}^{\infty} \exp \left(-\frac{\left(u-x e^{-\rho \Delta}\right)^{2}}{2 Q(\Delta)}\right) d u-\int_{-\infty}^{-y} \exp \left(-\frac{\left(u-x e^{-\rho \Delta}\right)^{2}}{2 Q(\Delta)}\right) d u\right) .
\end{aligned}
$$

Here, the second equality follows from the fact that $\mathrm{OU}$ process satisfies a reflection principle around zero, and the third inequality follows from the fact that $\left\{X_{\Delta}^{0} \leq-y\right\} \supset\left\{\tau_{0} \leq \Delta\right\}$ since $y>0$. The last line implies that (after taking the derivative with respect to $y$ and flipping the sign) the density of the random variable $Y_{\Delta}=X_{\Delta}^{0} 1_{\left\{\tau_{0}>\Delta\right\}}$ is given by

$$
q(y)=\frac{1}{\sqrt{2 \pi Q(\Delta)}}\left(\exp \left(-\frac{\left(y-x e^{-\rho \Delta}\right)^{2}}{2 Q(\Delta)}\right)-\exp \left(-\frac{\left(y+x e^{-\rho \Delta}\right)^{2}}{2 Q(\Delta)}\right)\right), \quad y>0 .
$$

Using (3.11) and (3.13), we can write

$$
B=\mathbb{E}^{x}\left[1_{\left\{\Delta<\tau_{0}\right\}} e^{-\alpha \Delta}\left(K\left(X_{\Delta}, a\right)+J^{\nu}(a)\right)\right]=e^{-\alpha \Delta}\left(\left(2 N\left(\frac{x e^{-\rho \Delta}}{\sqrt{Q(\Delta)}}\right)-1\right)\left(J^{\nu}(a)-a-\lambda\right)+A\right),
$$

in which

$$
A \triangleq \int_{0}^{\infty} y q(y) d y
$$

Since

$$
\int_{0}^{x e^{-\rho \Delta}} y \exp \left(-\frac{\left(y-x e^{-\rho \Delta}\right)^{2}}{2 Q(\Delta)}\right) d y=-\int_{-x e^{-\rho \Delta}}^{0} y \exp \left(-\frac{\left(y+x e^{-\rho \Delta}\right)^{2}}{2 Q(\Delta)}\right) d y,
$$

we can write $A$ as

$$
A=\frac{1}{\sqrt{2 \pi Q(\Delta)}}\left(\int_{x e^{-\rho \Delta}}^{\infty} y \exp \left(-\frac{\left(y-x e^{-\rho \Delta}\right)^{2}}{2 Q(\Delta)}\right) d y-\int_{-x e^{-\rho \Delta}}^{\infty} y \exp \left(-\frac{\left(y+x e^{-\rho \Delta}\right)^{2}}{2 Q(\Delta)}\right) d y\right) .
$$

For any $\mu \in \mathbb{R}$ and $\sigma>0$ we have that

$$
\int_{\mu}^{\infty} \frac{x}{\sqrt{2 \pi \sigma^{2}}} \exp \left(-\frac{(x-\mu)^{2}}{2 \sigma^{2}}\right) d x=\frac{\sigma}{\sqrt{2 \pi}}+\frac{\mu}{2} .
$$


As a result

$$
A=x e^{-\rho \Delta} \text {. }
$$

Observe from the above calculations that $X_{\Delta}^{0} 1_{\left\{\tau_{0}>\Delta\right\}}$ and $X_{\Delta}^{0}$ have the same expectation.

We will also compute the quantity

$$
\tilde{B} \triangleq \mathbb{E}^{x}\left[1_{\left\{\Delta<\tau_{0}\right\}} e^{-\alpha \Delta}\left(K\left(X_{\Delta}, a\right)+J^{\nu}(a)\right)-P 1_{\left\{\Delta>\tau_{0}\right\}} e^{-\alpha \tau_{0}}\right],
$$

for this case. Using the density of the hitting time of 0 , which can be derived by differentiating (3.11) we can write

$$
\tilde{B}=B-P \int_{0}^{\Delta} e^{-\alpha t} \frac{x}{\sqrt{2 \pi}}\left(\frac{\rho}{\sinh (\rho t)}\right)^{\frac{3}{2}} \exp \left(-\frac{\rho x^{2} e^{-\rho t}}{2 \sinh (\rho t)}+\frac{\rho t}{2}\right) d t .
$$

There is not explicit expression available for the integral term (even in terms of special functions, except when $\Delta=\infty$, see e.g. [6] and [8], in which case this integral is the Laplace transform of the distribution of $\tau_{0}$ ) but the NIntegrate function of Mathematica is able to evaluate it with a very high numerical precision.

Remark 3.1. We can compute $B$ in (3.8) explicitly even for the cases when $X^{0}$ follows

$$
d X_{t}^{0}=\left(\phi-\rho X_{t}^{0}\right) d t+\sigma d W_{t}, \quad X_{0}=x>0,
$$

for $\phi, \sigma>0$ by using the Strong Markov property to compute

$$
\mathbb{E}^{x}\left[X_{\Delta} 1_{\left\{\Delta<\tau_{0}\right\}}\right]=\mathbb{E}^{x}\left[X_{\Delta}\right]-\mathbb{E}^{x}\left[1_{\left\{\Delta \geq \tau_{0}\right\}} X_{\Delta}\right] .
$$

The Strong Markov property is used to compute

$$
\begin{aligned}
\mathbb{E}^{x}\left[1_{\left\{\Delta \geq \tau_{0}\right\}} X_{\Delta}\right] & =\mathbb{E}^{x}\left[1_{\left\{\Delta \geq \tau_{0}\right\}} \mathbb{E}^{x}\left[X_{\Delta} \mid \mathcal{F}_{\tau_{0}}\right]\right]=\mathbb{E}^{x}\left[1_{\left\{\Delta \geq \tau_{0}\right\}} \mathbb{E}^{0}\left[X_{\Delta-\tau_{0}}\right]\right] \\
& =\int_{0}^{\Delta} f(u) E^{0}\left[X_{\Delta-u}\right]=\phi \int_{0}^{\Delta} f(u)(1-\exp (-\rho(\Delta-u))) d u,
\end{aligned}
$$

where $f$ is the density function of $\tau_{0}$. Several representations for $f$ are available, see for e.g. [1].

3.1.2. Square-root Process. To evaluate $B$ in (3.8) when the aggregate income process is modeled by the square root process in (2.3) we need to compute

$$
C \triangleq \int_{0}^{\infty} y^{2} \tilde{q}(y) d y
$$

in which $\tilde{q}(y)$ is equal to the $q(y)$ in (3.13) if $x$ is replaced by $\sqrt{x}$. This follows because if (2.2) is started from $\sqrt{x}$, then the solution of it is the square root of the solution of (2.3). Let us first evaluate

$$
\begin{aligned}
& \frac{1}{\sqrt{2 \pi Q(\Delta)}} \int_{0}^{\infty} y^{2} \exp \left(-\frac{\left(y-\sqrt{x} e^{-\rho \Delta}\right)^{2}}{2 Q(\Delta)}\right) d y=\frac{1}{\sqrt{2 \pi}} \int_{-\frac{\sqrt{x} e^{-\rho \Delta}}{\sqrt{Q(\Delta)}}}^{\infty}\left(y \sqrt{Q(\Delta)}+\sqrt{x} e^{-\rho \Delta}\right)^{2} \exp \left(-\frac{y^{2}}{2}\right) d y \\
& =\frac{Q(\Delta)}{\sqrt{2 \pi}} \int_{-\frac{\sqrt{x} e^{-\rho \Delta}}{\sqrt{Q(\Delta)}}}^{\infty} y^{2} \exp \left(-\frac{y^{2}}{2}\right) d y+\frac{2 \sqrt{Q(\Delta)}}{\sqrt{2 \pi}} \sqrt{x} e^{-\rho \Delta} \int_{-\frac{\sqrt{x} e^{-\rho \Delta}}{\sqrt{Q(\Delta)}}}^{\infty} y \exp \left(-\frac{y^{2}}{2}\right) d y+x e^{-2 \rho \Delta} N\left(\frac{\sqrt{x} e^{-\rho \Delta}}{\sqrt{Q(\Delta)}}\right) \\
& =\frac{Q(\Delta)}{\sqrt{2 \pi}} \int_{-\frac{\sqrt{x} e^{-\rho \Delta}}{\sqrt{Q(\Delta)}}}^{\infty} y^{2} \exp \left(-\frac{y^{2}}{2}\right) d y+\frac{2 \sqrt{Q(\Delta)}}{\sqrt{2 \pi}} \sqrt{x} e^{-\rho \Delta} \exp \left(-\frac{x e^{-2 \rho \Delta}}{2 Q(\Delta)}\right)+x e^{-2 \rho \Delta} N\left(\frac{\sqrt{x} e^{-\rho \Delta}}{\sqrt{Q(\Delta)}}\right) .
\end{aligned}
$$


Since $\frac{1}{\sqrt{2 \pi Q(\Delta)}} \int_{0}^{\infty} y^{2} \exp \left(-\frac{\left(y+\sqrt{x} e^{-\rho \Delta}\right)^{2}}{2 Q(\Delta)}\right) d y$ can be obtained by flipping the sign in front of $\sqrt{x}$ in $(3.20)$, the computation of $C$ will follow. We also have that

$$
\begin{aligned}
& \frac{Q(\Delta)}{\sqrt{2 \pi}} \int_{-\frac{\sqrt{x}-\rho \Delta}{\sqrt{Q(\Delta)}}}^{\infty} y^{2} \exp \left(-\frac{y^{2}}{2}\right) d y=\frac{Q(\Delta)}{2}+\frac{Q(\Delta)}{\sqrt{2 \pi}} \int_{-\frac{\sqrt{x} e^{-\rho \Delta}}{\sqrt{Q(\Delta)}}}^{0} y^{2} \exp \left(-\frac{y^{2}}{2}\right) d y \\
& =\frac{Q(\Delta)}{2}+Q(\Delta) N\left(\frac{\sqrt{x} e^{-\rho \Delta}}{\sqrt{Q(\Delta)}}\right)-\frac{\sqrt{x Q(\Delta)}}{\sqrt{2 \pi}} e^{-\rho \Delta} \exp \left(-\frac{x e^{-2 \rho \Delta}}{2 Q(\Delta)}\right) .
\end{aligned}
$$

Form (3.20) and (3.21), we can evaluate $C$ as

$$
C=\left(x e^{-2 \rho \Delta}+Q(\Delta)\right)\left[2 N\left(\frac{\sqrt{x} e^{-\rho \Delta}}{\sqrt{Q(\Delta)}}\right)-1\right]+\frac{\sqrt{2 Q(\Delta) x}}{\sqrt{\pi}} e^{-\rho \Delta} \exp \left(-\frac{x e^{-2 \rho \Delta}}{Q(\Delta)}\right) .
$$

When $X^{0}$ is the square root process then $B$ defined in (3.8) equals

$$
B=e^{-\alpha \Delta}\left(\left(2 N\left(\frac{\sqrt{x} e^{-\rho \Delta}}{\sqrt{Q(\Delta)}}\right)-1\right)\left(J^{\nu}(a)-a-\lambda\right)+C\right) .
$$

3.1.3. Brownian Motion with Drift. Similarly, using reflection principle, Girsanov's Theorem and the spatial homogeneity of Brownian motion we will obtain $B$ in (3.14) when $X^{0}$ is a Brownian motion given by (2.1). We will first need the following lemma, which is Corollary B.3.4 in [19].

Lemma 3.1. Let $Y_{t}=\sigma W_{t}+\mu t$ and $m_{t}^{Y}=\min _{u \in[0, t]} Y_{u}$. Then

$$
\mathbb{P}\left\{Y_{t} \geq y, m_{t}^{Y} \geq m\right\}=N\left(\frac{-y+\mu t}{\sigma \sqrt{t}}\right)-e^{2 \mu m / \sigma^{2}} N\left(\frac{2 m-y-\mu t}{\sigma \sqrt{t}}\right)
$$

for every $m \leq 0$ and $y \geq m$.

We can write $B$ as

$$
B=\mathbb{E}^{x+\theta}\left[1_{\left\{\Delta<\tau_{\theta}\right\}} X_{\Delta}^{0}\right],
$$

in which $\theta \triangleq J^{\nu}(a)-a-\lambda$, which follows from the spatial homogeneity of Brownian motion. Note that for any $y>\theta$

$$
Z \triangleq 1_{\left\{\Delta<\tau_{\theta}\right\}} X_{\Delta}^{0} \geq y \quad \text { iff } \quad X_{\Delta} \geq y, m_{\Delta}^{X^{0}} \geq \theta .
$$

We will find the probability density function of Z. Let us first define

$$
Y_{t} \triangleq X_{t}-(\theta+x)
$$

which implies that $m_{t}^{Y}=m_{t}^{X^{0}}-(\theta+x)$. With this new definition

$\mathbb{P}\{Z \geq y\}=\mathbb{P}\left\{Y_{\Delta} \geq y-(x+\theta), m_{\Delta}^{Y} \geq-x\right\}=N\left(\frac{-y+x+\theta+\mu \Delta}{\sigma \sqrt{\Delta}}\right)-e^{-2 \mu x / \sigma^{2}} N\left(\frac{-x-y+\theta+\mu \Delta}{\sigma \sqrt{\Delta}}\right)$. 
Here, the second equality follows from Lemma 3.1. Now, the density of the random variable $Z$ is easy to calculate and using that we can compute $B$ by calculating the expectation of $Z$ and get

$$
\begin{aligned}
B & =e^{-\alpha \Delta}\left(\left(x+\mu \Delta+J^{\nu}(a)-a-\lambda\right) N\left(\frac{x+\mu \Delta}{\sigma \sqrt{\Delta}}\right)+\frac{\sigma \sqrt{\Delta}}{\sqrt{2 \pi}} \exp \left(-\frac{1}{2} \frac{(x+\mu \Delta)^{2}}{\sigma^{2} \Delta}\right)\right. \\
& \left.-e^{-2 \mu x / \sigma^{2}}\left(\left(-x+\mu \Delta+J^{\nu}(a)-a-\lambda\right) N\left(\frac{-x+\mu \Delta}{\sigma \sqrt{\Delta}}\right)+\frac{\sigma \sqrt{\Delta}}{\sqrt{2 \pi}} \exp \left(-\frac{1}{2} \frac{(-x+\mu \Delta)^{2}}{\sigma^{2} \Delta}\right)\right)\right) .
\end{aligned}
$$

3.1.4. Geometric Brownian Motion. We will use the down and out European call option price, see e.g. [24], (when we take the strike price to be zero) to evaluate

$$
\mathbb{E}^{x}\left[1_{\left\{\tau_{d}>\Delta\right\}} X_{\Delta}\right]=e^{\mu \Delta} x\left(N\left(d_{1}\right)-\left(\frac{d}{x}\right)^{1+2 \mu / \sigma^{2}} N\left(-d_{2}\right)\right),
$$

in which

$$
d_{1}=\frac{\log \frac{x}{d}+\left(\mu-\frac{1}{2} \sigma^{2}\right) \Delta}{\sigma \sqrt{\Delta}} \quad d_{2}=\frac{\log \frac{x}{d}-\left(\mu-\frac{1}{2} \sigma^{2}\right) \Delta}{\sigma \sqrt{\Delta}} .
$$

In order to calculate $B$ we also need to compute $\mathbb{P}\left\{\tau_{d}>\Delta\right\}$. In fact

$$
\mathbb{P}^{x}\left\{\tau_{d}>\Delta\right\}=\mathbb{P}^{x}\left\{\tau_{\tilde{d}}^{\tilde{B}}>\Delta\right\},
$$

in which $\tau_{\tilde{d}}^{\tilde{B}}$ is the hitting time of $\tilde{d}<0$ by the Brownian motion $\tilde{B} \triangleq \gamma t+\sigma B_{t}$, where

$$
\tilde{d} \triangleq \log \left(\frac{d}{x}\right), \quad \gamma=\mu-\frac{1}{2} \sigma^{2}
$$

Using the hitting time distribution for Brownian motion (with drift), (which can be obtained from Lemma 3.1), we deduce

$$
\mathbb{P}^{x}\left\{\tau_{d}>\Delta\right\}=\mathbb{P}^{x}\left\{m_{\Delta}^{\tilde{B}} \geq \tilde{d}\right\}=N\left(\frac{-\tilde{d}+\gamma t}{\sigma \sqrt{\Delta}}\right)-\exp \left(\frac{2 \gamma \tilde{d}}{\sigma^{2}}\right) N\left(\frac{\tilde{d}+\gamma t}{\sigma \sqrt{\Delta}}\right) .
$$

Therefore, $B$ can be written as

$$
B=e^{-\alpha \Delta}\left[e^{\mu \Delta} x\left(N\left(d_{1}\right)-\left(\frac{d}{x}\right)^{1+2 \mu / \sigma^{2}} N\left(-d_{2}\right)\right)+\left(J^{\nu}(a)-a-\lambda\right) \mathbb{P}^{x}\left\{\tau_{d}>\Delta\right\}\right] .
$$

\section{An Efficient Algorithm to Calculate the Value Function}

4.1. Increasing and Decreasing Solutions of $(\mathcal{A}-\alpha) u=0$. When $X^{0}$ is the Brownian motion in (2.1), then the increasing and decreasing solutions of $(\mathcal{A}-\alpha) u=0$ are

$$
\psi(x)=e^{D_{1} x} \quad \text { and } \quad \varphi(x)=e^{D_{2} x}
$$

in which

$$
D_{1}=\frac{-\mu+\sqrt{\mu^{2}+2 \alpha \sigma^{2}}}{\sigma^{2}} \text { and } D_{2}=\frac{-\mu-\sqrt{\mu^{2}+2 \alpha \sigma^{2}}}{\sigma^{2}} .
$$

When $X^{0}$ is the Ornstein-Uhlenbeck process in (2.2), then

$$
\psi(x)=\exp \left(\frac{\rho x^{2}}{2}\right) D_{-\alpha / \rho}(-x \sqrt{2 \rho}), \quad \varphi(x)=\exp \left(\frac{\rho x^{2}}{2}\right) D_{-\alpha / \rho}(x \sqrt{2 \rho}), \quad x \in \mathbb{R},
$$


where $D_{\nu}(\cdot)$ is the parabolic cylinder function given in the Appendices 1.14 and 2.9 in [6] which is defined as

$$
D_{\nu}(x) \triangleq 2^{-\nu / 2} e^{-x^{2} / 4} H_{v}\left(\frac{x}{\sqrt{2}}\right), \quad x \in \mathbb{R},
$$

in which $H_{\nu}$ is the Hermite polynomial of order $\nu$, which has the integral representation (see e.g. [17])

$$
H_{\nu}(x)=\frac{1}{\Gamma(-\nu)} \int_{0}^{\infty} \exp \left(-t^{2}-2 t x\right) t^{-\nu-1} d t, \quad \operatorname{Re}(\nu)<0 .
$$

On the other hand, when $X^{0}$ is the square root process whose dynamics follows (2.3), then

$$
\psi(x)=x^{-1 / 4} \exp \left(\frac{\rho x}{2}\right) M_{-\frac{\alpha}{2 \rho}+\frac{1}{4},-\frac{1}{4}}(\rho x), \quad \varphi(x)=x^{-1 / 4} \exp \left(\frac{\rho x}{2}\right) W_{-\frac{\alpha}{2 \rho}+\frac{1}{4},-\frac{1}{4}}(\rho x),
$$

in which $W_{-\frac{\alpha}{2 \rho}+\frac{1}{4},-\frac{1}{4}}$ and $M_{-\frac{\alpha}{2 \rho}+\frac{1}{4},-\frac{1}{4}}$ are Whittaker functions (see e.g. Appendix 2.10 of [6]). These functions satisfy

$$
\begin{aligned}
& W_{-\frac{\alpha}{2 \rho}+\frac{1}{4},-\frac{1}{4}}\left(\frac{x^{2}}{2}\right)=2^{\frac{\alpha}{2 \rho}-\frac{1}{4}} \sqrt{x} D_{-\alpha / \rho}(x), \quad x \geq 0, \\
& M_{-\frac{\alpha}{2 \rho}+\frac{1}{4},-\frac{1}{4}}\left(\frac{x^{2}}{2}\right)=\frac{\Gamma((1+\alpha / \rho) / 2)}{2 \sqrt{\pi}} \sqrt{x}\left(D_{-\alpha / \rho}(-x)-D_{-\alpha / \rho}(x)\right), \quad x \geq 0,
\end{aligned}
$$

in which $\Gamma$ stands for the Gamma function $\Gamma(x)=\int_{0}^{\infty} u^{x-1} e^{-u} d u$. When, $X^{0}$ is the geometric Brownian motion, then

$$
\psi(x)=x^{\sqrt{\kappa^{2}+\frac{2 \alpha}{\sigma^{2}}}-\kappa} \quad \varphi(x)=x^{-\sqrt{\kappa^{2}+\frac{2 \alpha}{\sigma^{2}}}-\kappa},
$$

in which $\kappa=\mu / \sigma^{2}-1 / 2$.

4.2. An Algorithm to Find the Optimal Control. In this section we will describe a numerical algorithm to find the value function. First we will introduce some notation that we facilitate our description.

$$
e^{\alpha \Delta} B=: r(x ; a)+h(x) J^{\nu}(a),
$$

where $B$ is as in (3.8). We transform $r$ and $h$ by

$$
R(\cdot ; a) \triangleq \frac{r\left(F^{-1}(\cdot), a\right)}{\varphi\left(F^{-1}(\cdot)\right)}, \quad H(\cdot) \triangleq \frac{h\left(F^{-1}(\cdot)\right)}{\varphi\left(F^{-1}(\cdot)\right)} .
$$

Note that $r(a ; a)<0$ and that $\sup _{x} r(x ; a)>0$ in all the cases considered above (see Section 4.3). First stage: For a given pair $(a, b) \in \mathbb{R}_{+}^{2}$ we will determine $W$ in (3.6) using the linear characterization in (3.7). On $[F(0), F(b)]$ we will find the line $W(y)=\beta y+\xi$ that passes through the point $\left(F(0),-\frac{P}{\varphi(0)}\right)$, i.e.,

$$
\xi=-\beta F(0)-\frac{P}{\varphi(0)}
$$

and satisfies

$$
\beta F(b)+\xi=e^{-\alpha \Delta}[R(F(b) ; a)+H(F(b)) \varphi(a)(\beta F(a)+\xi)] .
$$


$P=0$ when we consider (3.3). The slope $\beta$ can be determined as

$$
\beta=\frac{\frac{P}{\varphi(0)}+e^{-\alpha \Delta}\left[R(F(b) ; a)-H(F(b)) \varphi(a) \frac{P}{\varphi(0)}\right]}{F(b)-F(a)-e^{-\alpha \Delta}(F(a)-F(0)) H(F(b)) \varphi(a)} .
$$

Now the function $J^{\nu}(x)$ in $(3.4)$ can be written as

$$
J^{\nu}(x)= \begin{cases}\beta \psi(x)+\xi \varphi(x), & 0 \leq x \leq b, \\ e^{-\alpha \Delta}\left(r(x ; a)+h(x) J^{\nu}(a)\right), & x \geq b .\end{cases}
$$

Note that $(\mathcal{A}-\alpha) J^{\nu}(x)=0$ for $x<b$.

Second stage: Let us fix $a$ and treat $\beta$ as a function of $b$ parametrized by $a$. We will maximize the function $\beta$ in (4.10). Taking the derivative of (4.9) and evaluating at $\beta_{b}=0$ we obtain

$$
\beta=e^{-\alpha \Delta}\left[\left.\frac{\partial}{\partial y} R(y ; a)\right|_{y=F(b)}+H^{\prime}(F(b)) \varphi(a)\left(\beta \cdot(F(a)-F(0))-\frac{P}{\varphi(0)}\right)\right],
$$

in which $\beta$ is as in (4.10). To find the optimal $b$ given $a$ we solve the non-linear and implicit equation (4.12).

Remark 4.1. On $y \geq F(b)$, the function $W$ is given by

$$
W(y)=e^{-\alpha \Delta}[W(F(a)) \varphi(a) H(y)+R(y ; a)] .
$$

The right derivative of $W$ at $F(b)$ is

$$
W^{\prime}(F(b))=e^{-\alpha \Delta}\left(W(F(a)) \varphi(a) H^{\prime}(F(b))+\left.\frac{\partial}{\partial y} R(y ; a)\right|_{y=F(b)}\right)=\beta,
$$

where we used (4.12). This implies that the left and the right derivatives of $W$ are equal at $F(b)$ (smooth fit), since the left derivative at $F(b)$ is also equal to $\beta$.

Third stage: Now, we vary $a \in \mathbb{R}_{+}$and choose $a^{*}$ that maximizes $a \rightarrow \beta(a)$. We also find the corresponding $b^{*}=b\left(a^{*}\right)$. Now, the value function is given by (4.11) when $a$ and $b$ are replaced by $a^{*}$ and $b^{*}$ respectively.

The next proposition justifies the second stage of our algorithm.

Proposition 4.1. Assume that $r(a ; a)<0$ and $\sup _{x} r(x ; a)>0$. Furthermore, if the functions $R(\cdot ; a)$ and $H(\cdot)$ defined in (4.7) are increasing and concave on $(y, \infty)$ for some $y \geq F(a)$, and the function $h(\cdot)$ defined in (4.6) satisfies $h(\cdot) \in(0,1)$, then for any given $a \geq 0$, (4.12) has a unique solution.

The proof essentially follows from Remark 4.1. But we will have to introduce a series of lemmas before we justify our claim.

First, let us also introduce a family of value functions parameterized by $\gamma \in \mathbb{R}$ as

$$
V_{a}^{\gamma}(x) \triangleq \sup _{\tau \in \mathcal{S}} \mathbb{E}^{x}\left[e^{-\alpha \tau} r^{\gamma}\left(X_{\tau} ; a\right)\right] \quad \text { where } r^{\gamma}(x ; a) \triangleq e^{-\alpha \Delta}(r(x ; a)+\gamma \cdot h(x)),
$$

in which $\mathcal{S}$ is the set of stopping times of the natural filtration of $X$. Here, $X$ is a diffusion on $[0, \infty)$, which is absorbed at the left boundary. (In the case of geometric Brownian motion this left boundary is taken to be $d>0$.) Then we have the following result. 
Lemma 4.2. Let us define

$$
R^{\gamma}(\cdot ; a) \triangleq \frac{r^{\gamma}\left(F^{-1}(\cdot), a\right)}{\varphi\left(F^{-1}(\cdot)\right)}
$$

then the function

$$
W_{a}^{\gamma}(\cdot) \triangleq \frac{V_{a}^{\gamma}\left(F^{-1}(\cdot)\right)}{\varphi\left(F^{-1}(\cdot)\right)},
$$

is the smallest non-negative concave majorant of $R^{\gamma}$ that passes through $(F(0), 0)$. Moreover under the assumptions of Proposition 4.1 this majorant is linear in the continuation region (the region in which $W^{\gamma}$ is strictly greater than $R^{\gamma}$ ).

Proof. The first part of the proof follows Proposition 5.3 of Dayanik and Karatzas [9]. The second part of the proof follows from the first and the fact that $R^{\gamma}(\cdot ; a)$ is increasing and concave on $(y, \infty)$.

The following technical lemma will be used in showing the existence of $\gamma$ such that $V_{a}^{\gamma}(a)=\gamma$ for any $a \geq 0$.

\section{Lemma 4.3.}

$$
V_{a}^{\gamma_{1}}(x)-V_{a}^{\gamma_{2}}(x) \leq \gamma_{1}-\gamma_{2}, \quad \text { for any } \quad \gamma_{1} \geq \gamma_{2} \quad \text { and } \quad x \geq 0
$$

Proof. It is clear from (4.6) that $\gamma \rightarrow V_{a}^{\gamma}(x)$ is an increasing convex function. Therefore the rightderivative

$$
D_{\gamma}^{+} V_{a}^{\gamma^{\prime}}(x) \triangleq \lim _{h \downarrow 0} \frac{V^{\gamma^{\prime}+h}(x)-V^{\gamma^{\prime}}(x)}{h}
$$

exists for any $\gamma^{\prime}>0$ and it satisfies

$$
\frac{V_{a}^{\gamma_{1}}(x)-V_{a}^{\gamma_{2}}(x)}{\gamma_{1}-\gamma_{2}} \leq D_{\gamma}^{+} V_{a}^{\gamma_{1}}(x)
$$

for any $\gamma_{1} \geq \gamma_{2}$ (see e.g. [15], pages 213-214). Note that since $h(\cdot) \in(0,1)$ we have that

$$
0<D_{\gamma}^{+} V_{a}^{\gamma^{\prime}}(x) \leq 1
$$

Now, (4.19) and (4.20) together imply (4.18).

Lemma 4.4. Under the assumptions of Proposition 4.1, there exists a unique $\gamma$ such that $V_{a}^{\gamma}(a)=\gamma$ for $a \geq 0$.

Proof. Consider the function $\gamma \rightarrow V_{a}^{\gamma}(a)$. Our aim is to show that there exists a fixed point to this function. Let us consider $V_{a}^{0}(a)$ first. Since $\sup _{x} r(x ; a)>0$ we have that $V_{a}^{0}(a)>0$. Now let us consider the case when $\gamma>0$. First, note that $W_{a}^{\gamma}(F(a)) \geq R^{\gamma}(F(a), a)$ for all $\gamma$. Since by Lemma 4.3 $V$ has less than linear growth in $\gamma$ and $R^{\gamma}$ is linear in $\gamma$, we can find a $\gamma^{\prime}$ large enough such that $W_{a}^{\gamma}(F(a))=R^{\gamma}(F(a), a)$ for $\gamma \geq \gamma^{\prime}$. This implies however

$$
V_{a}^{\gamma^{\prime}}(a)=\varphi(a) R^{\gamma^{\prime}}(F(a) ; a)=e^{-\alpha \Delta}\left(r(a ; a)+\gamma^{\prime} h(a)\right)<\gamma^{\prime} .
$$

Since $\gamma \rightarrow V_{a}^{\gamma}$ is continuous, which follows from the fact that this function is convex, $V_{a}^{0}>0$ and $V_{a}^{\gamma^{\prime}}(a)<\gamma^{\prime}$ implies that $\gamma \rightarrow V_{a}^{\gamma}$ crosses the line $\gamma \rightarrow \gamma$. Since $\gamma \rightarrow V_{a}^{\gamma}$ is increasing convex it crosses this line only once. 
Proof of Proposition 4.1. The smallest concave majorant $W_{a}^{\gamma}$ in $(4.17)$ is linear on $\left(F(0), F\left(b^{\gamma}\right)\right)$ for a unique $b^{\gamma} \in[0, \infty)$ and smoothly fits to $R^{\gamma}(\cdot ; a)$ at $b^{\gamma}$ and coincides with $R^{\gamma}(\cdot, a)$ on $\left[b^{\gamma}\right.$, d). Together with Lemma 4.4 this implies that there exists a unique $\gamma^{*}$ such that equations (4.13) and (4.14) are satisfied when $W$ is replaced by $W_{a}^{\gamma^{*}}$ and $b$ is replaced by $b^{\gamma^{*}}$. If the solution of equations (4.13) and (4.14) were not unique, on the other hand, then one would be able to find multiple smooth fit points $b^{\gamma^{*}}$, which yields a contradiction.

4.3. Are the Assumptions of Proposition 4.1 Satisfied? The following remark will be helpful in the analysis that follows:

Remark 4.2. Given a function $k$ let us denote $K(y) \triangleq \frac{k}{\varphi} \circ F^{-1}(y), y>0$. If $k$ is twice differentiable at $x \geq 0$ and if we denote $y \triangleq F(x)$, then $K^{\prime}(y)=m(x)$ and $K^{\prime \prime}(y)=\frac{m^{\prime}(x)}{F^{\prime}(x)}$ with

$$
m(x)=\frac{1}{F^{\prime}(x)}\left(\frac{k}{\varphi}\right)^{\prime}(x), \quad \text { and } \quad K^{\prime \prime}(y)[(\mathcal{A}-\alpha) k(x)] \geq 0, \quad y=F(x),
$$

with strict inequality if $H^{\prime \prime}(y) \neq 0$. The inequality in (4.21) is useful in identifying the concavity of $K$.

4.3.1. Brownian Motion with Drift. In this case $r(x ; a)$ and $h(x)$ defined in $(4.6)$ are given by

$$
\begin{aligned}
r(x ; a) & =(x+\mu \Delta-a-\lambda) N\left(\frac{x+\mu \Delta}{\sigma \sqrt{\Delta}}\right)+\sigma \sqrt{\Delta} \phi\left(\frac{x+\mu \Delta}{\sigma \sqrt{\Delta}}\right) \\
& -e^{-2 \mu x / \sigma^{2}}\left((-x+\mu \Delta-a-\lambda) N\left(\frac{-x+\mu \Delta}{\sigma \sqrt{\Delta}}\right)+\sigma \sqrt{\Delta} \phi\left(\frac{-x+\mu \Delta}{\sigma \sqrt{\Delta}}\right)\right), \\
h(x) & =\left(N\left(\frac{x+\mu \Delta}{\sigma \sqrt{\Delta}}\right)-e^{-2 \mu x / \sigma^{2}} N\left(\frac{-x+\mu \Delta}{\sigma \sqrt{\Delta}}\right)\right)
\end{aligned}
$$

in which $\phi(x)=(1 / \sqrt{2 \pi}) e^{-x^{2} / 2}$.

First note that $h(x) \in(0,1)$. It is enough to show that $R(\cdot ; a)$ and $H(\cdot)$ are eventually increasing, and are eventually concave. First, we will show that they are eventually increasing. The derivative of $R(\cdot ; a)$ has the same sign as

$$
\begin{aligned}
\varphi(x)^{2}\left(\frac{r(x ; a)}{\varphi(x)}\right)^{\prime}(x) & =\varphi(x)\left\{N\left(\frac{x+\mu \Delta}{\sigma \sqrt{\Delta}}\right)-\frac{(a+\lambda)}{\sigma \sqrt{\Delta}} \phi\left(\frac{x+\mu \Delta}{\sigma \sqrt{\Delta}}\right)\right. \\
& +e^{-2 \mu x / \sigma^{2}}\left(N\left(\frac{-x+\mu \Delta}{\sigma \sqrt{\Delta}}\right)-\frac{(a+\lambda)}{\sigma \sqrt{\Delta}} \phi\left(\frac{-x+\mu \Delta}{\sigma \sqrt{\Delta}}\right)\right) \\
& -D_{1} \sigma^{2}\left((-x+\mu \Delta-a-\lambda) N\left(\frac{-x+\mu \Delta}{\sigma \sqrt{\Delta}}\right)+\sigma \sqrt{\Delta} \phi\left(\frac{-x+\mu \Delta}{\sigma \sqrt{\Delta}}\right)\right) \\
& \left.-D_{2} e^{-2 \mu x / \sigma^{2}}\left((x+\mu \Delta-a-\lambda) N\left(\frac{x+\mu \Delta}{\sigma \sqrt{\Delta}}\right)+\sigma \sqrt{\Delta} \phi\left(\frac{x+\mu \Delta}{\sigma \sqrt{\Delta}}\right)\right)\right\},
\end{aligned}
$$

since $F$ is an increasing function. If we take $x>a$ ( $a$ is fixed) large enough, the third line of (4.23) dominates the the other lines. Since $D_{1}>0$, we can conclude that there exists $x^{\prime} \geq a$ such that $\left(\frac{r(x ; a)}{\varphi(x)}\right)^{\prime}(x)>0$ on $x \in\left(x^{\prime}, \infty\right)$. 
On the other hand, directly taking the derivative, $h(x)$ can be shown to be an increasing function in $x \in \mathbb{R}_{+}$, from which it follows that $H(y)=h\left(F^{-1}(y)\right) / \varphi\left(F^{-1}(y)\right)$ is also increasing.

Next, we will show that $R$ and $H$ are eventually concave. Consider the equation $(\mathcal{A}-\alpha) r(x ; a)=p(x ; a)$ so that

$$
p(x ; a)=\mu r^{\prime}(x ; a)+\frac{1}{2} \sigma^{2} r^{\prime \prime}(x ; a)-\alpha r(x ; a) .
$$

Directly taking the derivatives and letting $x \rightarrow \infty$, we obtain $r^{\prime}(x ; a) \rightarrow 1, r^{\prime \prime}(x ; a) \rightarrow 0$ and $r(x ; a) \rightarrow \infty$. Therefore $\lim _{x \rightarrow \infty} p(x ; a)=-\infty$. Similarly, we consider the equation $q(x) \triangleq(\mathcal{A}-\alpha) h(x)$. By letting $x \rightarrow \infty$, we have $h(x) \rightarrow 1, h^{\prime}(x) \rightarrow 0$ and $h^{\prime \prime}(x) \rightarrow 0$ so that $\lim _{x \rightarrow \infty} q(x)<0$. Together with Remark 4.2, these facts imply that $R(\cdot, a)$ and $H(\cdot)$ are concave on $y \in\left(y^{\prime \prime},+\infty\right)$ for some $y^{\prime \prime} F(a)$.

4.3.2. Ornstein-Uhlenbeck Process. We will only consider the case when the performance function is as in (3.3). The analysis for the case when declaring banktruptcy is penalized can be performed similarly, since first and the second derivatives of the integral term in (3.17) with respect to the $x$ variable goes to zero as $x \rightarrow \infty$.

In this case $r(x ; a)$ and $h(x)$ defined in (4.6) are given by

$$
r(x ; a)=x e^{-\rho \Delta}-\left(2 N\left(\frac{x e^{-\rho \Delta}}{\sqrt{Q(\Delta)}}\right)-1\right)(a+\lambda), \quad h(x)=2 N\left(\frac{x e^{-\rho \Delta}}{\sqrt{Q(\Delta)}}\right)-1 .
$$

First, observe that $r(x ; a)>0$ on $\left(x_{1}, \infty\right)$ with some $x_{1}>a$. By taking the derivative of $r(x ; a)$, we have

$$
r^{\prime}(x ; a)=e^{-\rho \Delta}\left(1-\frac{2(a+\lambda)}{\sqrt{Q(\Delta)}} \phi\left(\frac{x e^{-\rho \Delta}}{\sqrt{Q(\Delta)}}\right)\right) .
$$

From this expression we see that $r^{\prime}(x ; a)>0$ on $x \in\left(x_{2}, \infty\right)$ with some $x_{2}>a$. Let us denote $x^{\prime} \triangleq$ $\max \left(x_{1}, x_{2}\right)$. It follows that $R(y)$ is increasing on $y \in\left(y^{\prime}, \infty\right)$ with $y^{\prime}=F\left(x^{\prime}\right)$ because

$$
\left(\frac{r}{\varphi}\right)^{\prime}=\frac{r^{\prime} \varphi-r \varphi^{\prime}}{\varphi^{2}} \quad \text { with } \quad \varphi^{\prime}<0 .
$$

Observe also that $h(x) \in(0,1)$ and $h^{\prime}(x)>0$ on $x \in \mathbb{R}_{+}$.

Next, we will analyze the concavity properties of $R(\cdot ; a)$ and $H$. Consider the equation $(\mathcal{A}-\alpha) r(x ; a)=$ $p(x ; a)$ so that

$$
p(x ; a)=-\rho x r^{\prime}(x ; a)+\frac{1}{2} r^{\prime \prime}(x ; a)-\alpha r(x ; a) .
$$

We have $r(x ; a) \rightarrow+\infty, x^{\prime}(x ; a) \rightarrow+\infty$ and $r^{\prime \prime}(x ; a) \rightarrow 0$ as $x \rightarrow \infty$. Thus, we have $\lim _{x \rightarrow \infty} p(x ; a)=$ $-\infty$. Similarly, we consider the equation $q(x) \triangleq(\mathcal{A}-\alpha) h(x)$. By letting $x \rightarrow \infty$, we have $h(x) \rightarrow 1$, $x h^{\prime}(x) \rightarrow 0$ and $h^{\prime \prime}(x) \rightarrow 0$ so that $\lim _{x \rightarrow \infty} q(x)<0$. Together with Remark 4.2, this analysis shows that there exists $y^{\prime \prime} \geq F(a)$ such that $R(\cdot ; a)$ and $H(\cdot)$ are concave on $\left(y^{\prime \prime}, \infty\right)$.

4.3.3. Square Root Process. In this case the functions $r$ and $h$ are given by

$$
\begin{aligned}
r(x ; a) & =\left(x e^{-2 \rho \Delta}+Q(\Delta)-(a+\lambda)\right)\left(2 N\left(\frac{\sqrt{x} e^{-\rho \Delta}}{\sqrt{Q(\Delta)}}\right)-1\right)+2 \sqrt{Q(\Delta) x} e^{-\rho \Delta} \phi\left(\frac{\sqrt{x} e^{-\rho \Delta}}{\sqrt{Q(\Delta)}}\right), \\
h(x) & =2 N\left(\frac{\sqrt{x} e^{-\rho \Delta}}{\sqrt{Q(\Delta)}}\right)-1 .
\end{aligned}
$$


Observe that $r(x ; a)>0$ on $\left(x_{1}, \infty\right)$ with some $x_{1} \geq a$ since the only negative term in the first equation in (4.25) is bounded from below by $-(a+\lambda)$. Taking the derivative of $r(x ; a)$ we obtain

$$
\begin{aligned}
r^{\prime}(x ; a) & =e^{-2 \rho \Delta}\left(2 N\left(\frac{\sqrt{x} e^{-\rho \Delta}}{\sqrt{Q(\Delta)}}\right)-1\right)+\frac{e^{-\rho \Delta}}{\sqrt{Q(\Delta)}} \phi\left(\frac{\sqrt{x} e^{-\rho \Delta}}{\sqrt{Q(\Delta)}}\right)\left(\sqrt{x} e^{-2 \rho \Delta}+\frac{a+\lambda}{\sqrt{x}}\right) \\
& -\frac{e^{-\rho \Delta}}{\sqrt{x}} \phi\left(\frac{\sqrt{x} e^{-\rho \Delta}}{\sqrt{Q(\Delta)}}\right)\left(\sqrt{Q(\Delta)}+\frac{x e^{-\rho \Delta}+Q(\Delta)}{\sqrt{Q(\Delta)}}\right) .
\end{aligned}
$$

The second term on the first line of (4.26) is positive and it dominates as $x \rightarrow \infty$, therefore $r^{\prime}(x ; a)>0$ on $x \in\left(x_{2}, \infty\right)$ with for some $x_{2} \geq a$. Take $x^{\prime} \triangleq \max \left(x_{1}, x_{2}\right)$. It follows that $R(y)$ is increasing on $y \in\left(y^{\prime}, \infty\right)$, in which $y^{\prime}=F\left(x^{\prime}\right)$. On the other hand, $h(x) \in(0,1)$ and $h^{\prime}(x)=-\frac{e^{-\rho \Delta}}{\sqrt{x}} \phi\left(\frac{\sqrt{x} e^{-\rho \Delta}}{\sqrt{Q(\Delta)}}\right)<0$. However, $h^{\prime}$ goes to zero as $x \rightarrow \infty$, which implies that $(h / \varphi)^{\prime}=\frac{h^{\prime} \varphi-h \varphi^{\prime}}{\varphi^{2}}>0$ on $\left(x^{\prime \prime}, \infty\right)$ for some sufficiently large $x^{\prime \prime}$.

Next, we analyze the concavity properties of $R(\cdot ; a)$ and $H(\cdot)$. Let us define $p(x ; a) \triangleq(\mathcal{A}-\alpha) r(x ; a)$. As a result

$$
p(x ; a)=(1-2 \rho x) r^{\prime}(x ; a)+2 x r^{\prime \prime}(x ; a)-\alpha r(x ; a) .
$$

We have $r(x ; a) \rightarrow+\infty, x r^{\prime}(x ; a) \rightarrow+\infty$ and $x r^{\prime \prime}(x ; a) \rightarrow 0$ as $x \rightarrow \infty$. Thus, we have $\lim _{x \rightarrow \infty} p(x ; a)=$ $-\infty$. Similarly, we consider the equation $q(x) \triangleq(\mathcal{A}-\alpha) h(x)$. By letting $x \rightarrow \infty$, we have $h(x) \rightarrow 1$, $x h(x) \rightarrow 0$ and $x h^{\prime \prime}(x) \rightarrow 0$ so that $\lim _{x \rightarrow \infty} q(x)<0$. Using Remark 4.2, we observe that $R(\cdot ; a)$ and $H(\cdot)$ are eventually concave.

4.3.4. Geometric Brownian Motion. When the aggregate income process $X^{0}$ is modeled by a geometric Brownian motion a sufficient condition for the hypothesis of the Proposition 4.1 to hold is $\mu \leq \alpha$. In this case the functions $r$ and $h$ are given by

$$
\begin{aligned}
r(x ; a) & =e^{\mu \Delta} x\left(N\left(d_{1}\right)-\left(\frac{d}{x}\right)^{1+2 \mu / \sigma^{2}} N\left(-d_{2}\right)\right)-(a+\lambda)\left(N\left(\frac{-\tilde{d}+\gamma \Delta}{\sigma \sqrt{\Delta}}\right)-e^{2 \gamma \tilde{d} / \sigma^{2}} N\left(\frac{\tilde{d}+\gamma \Delta}{\sigma \sqrt{\Delta}}\right)\right), \\
h(x) & =\left(N\left(\frac{-\tilde{d}+\gamma \Delta}{\sigma \sqrt{\Delta}}\right)-e^{2 \gamma \tilde{d} / \sigma^{2}} N\left(\frac{\tilde{d}+\gamma \Delta}{\sigma \sqrt{\Delta}}\right)\right) .
\end{aligned}
$$

Observe that $\tilde{d}<0$ since $x>d$. Moreover, $N\left(d_{1}\right) \rightarrow 1, N\left(-d_{2}\right) \rightarrow 0$ and $N(\tilde{d}) \rightarrow 0$ as $x \rightarrow+\infty$. Also, $r(x ; a)>0$ on $\left(x_{1}, \infty\right)$ with some $x_{1}>a$ since the negative term in the first equation in (4.27) is bounded. On the other hand $h(x)>0$ for $x \in \mathbb{R}_{+}$and $h^{\prime}(x) \rightarrow 0$ as $x \rightarrow \infty$. The derivative of $r$ is

$$
\begin{aligned}
r^{\prime}(x ; a) & =e^{\mu \Delta}\left(N\left(d_{1}\right)-\left(\frac{d}{x}\right)^{1+2 \mu / \sigma^{2}} N\left(-d_{2}\right)\right)-(a+\lambda) h^{\prime}(x) \\
& +e^{\mu \Delta}\left(\frac{\phi\left(d_{1}\right)}{\sigma \sqrt{\Delta}}+\left(1+2 \mu / \sigma^{2}\right)\left(\frac{d}{x}\right)^{1+2 \mu / \sigma^{2}}+\left(\frac{\phi\left(-d_{2}\right)}{\sigma \sqrt{\Delta}}\right)\left(\frac{d}{x}\right)^{1+2 \mu / \sigma^{2}}\right),
\end{aligned}
$$


which is positive on $x \in\left(x_{2}, \infty\right)$ for some $x_{2} \geq a$. Take $x^{\prime} \triangleq \max \left(x_{1}, x_{2}\right)$. It follows that $R(y)$ is increasing on $y \in\left(y^{\prime}, \infty\right)$ with $y^{\prime}=F\left(x^{\prime}\right)$. Similarly, since $h(x) \in(0,1)$ and $h^{\prime}(x)$ goes to zero as $x \rightarrow \infty$, so that $H^{\prime}(y)>0$ on $\left(y^{\prime \prime}, \infty\right)$ for sufficiently large $y^{\prime \prime}$.

Next, we analyze the concavity of $R(\cdot ; a)$ and $H(\cdot)$. Let us denote $p(x ; a) \triangleq(\mathcal{A}-\alpha) r(x ; a)$. The function $p(\cdot ; a)$ is given by

$$
\begin{aligned}
p(x ; a) & =\mu x r^{\prime}(x ; a)+\frac{1}{2} \sigma^{2} r^{\prime \prime}(x ; a)-\alpha r(x ; a) \\
& =(\mu-\alpha) x e^{\mu \Delta}\left(N\left(d_{1}\right)-\left(\frac{d}{x}\right)^{1+2 \mu / \sigma^{2}} N\left(-d_{2}\right)\right)-\alpha(a+\lambda) h(x)+T(x ; a),
\end{aligned}
$$

where $T(x ; a)$ is the terms that involve $\phi(\cdot)$ or $\left(\frac{d}{x}\right)^{1+2 \mu / \sigma^{2}}$ and $\lim _{x \rightarrow+\infty} T(x ; a)=0$. Observe that $\lim _{x \rightarrow+\infty} p(x ; a)=-\infty$ when $\mu \leq \alpha$. Similarly, we consider the equation $q(x) \triangleq(\mathcal{A}-\alpha) h(x) . h(x) \rightarrow 1$, $h^{\prime}(x) \rightarrow 0$ and $h^{\prime \prime}(x) \rightarrow 0$ implies that $\lim _{x \rightarrow \infty} q(x)<0$. Using Remark 4.2, we can conclude that $R(\cdot ; a)$ and $H(\cdot)$ are eventually concave.

\section{Numerical Examples}

See Figures 1-4 for numerical illustrations. In our examples we quantify the effect of delay in dividend payments. In each case we find the optimal dividend payment barrier, $b^{*}$, the optimal amount of dividend payment, $b^{*}-a^{*}$, and the value function $v$. Then we compare them to $b^{0}, b^{0}-a^{0}$ and $v^{0}$, the analogues of the previous quantities when there is no delay. As expected the value function is smaller, $v<v_{0}$ when there is delay in dividend payments. Since in Figures 2 (b), 2 (e) and 4 (b), the value functions $v$ and $v_{0}$ are not distinguishable, in Figures (2) and (4) we plot the difference of $v_{0}-v$.

When the aggregate income process, $X^{0}$ is modeled by a Brownian motion with drift, a square root process then we observe that $a^{*}<a_{0}, b^{*}<b_{0}, b^{*}-a^{*}<b_{0}-a_{0}$ and $\beta^{*}<\beta_{0}$. The same conclusion holds if $X^{0}$ is an Ornstein-Uhlenbeck process and the declaring bankruptcy is penalized. On the other hand, when $X^{0}$ is modeled by an Ornstein-Uhlenbeck process (the case in which declaring ruin is not penalized) or a geometric Brownian motion we obtain that $a^{*}=a_{0}, b^{*}>b_{0}, b^{*}-a^{*}>b_{0}-a_{0}$ and $\beta^{*}<\beta_{0}$. Note that in both of these cases declaring bankruptcy is optimal as soon as the aggregate income level hits $b^{*}$, regardless of the magnitude of delay.

Observe that in the numerical examples considered, the function $\beta(a)$, which is obtained from (4.10) after we plug in for $b$ that we obtain from (4.12) (say $b(a)$ ), is concave. It is either strictly decreasing or has a unique local maximum. We leave the proof of these features of the function $\beta(a)$ as an open problem.

Remark 5.1. In our framework, it is easy to deal with solvency constraints. The optimal $a^{*}$ may not be acceptable, and prohibited by regulatory constraints. This was studied by Paulsen [22] in singular control setting (with no delays). Let as consider the case with $\Delta=0$ and assume that the firm is not allowed to reduce its aggregate cash flow to below $\tilde{a}$. If we show the above properties hold for $\beta(a) a$ it is easy to argue if $a^{*}>\tilde{a}$, then every time it pays out dividends the firm would reduce its reservoir to $a^{*}$ (the constraint is not binding), else if $a^{*}<\tilde{a}$, then the firm every time it pays out dividends the firm would reduce its reservoir to $\tilde{a}$. 


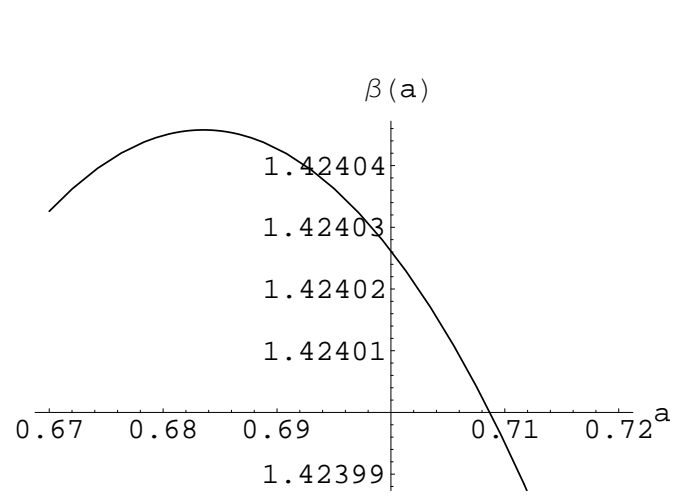

(a)

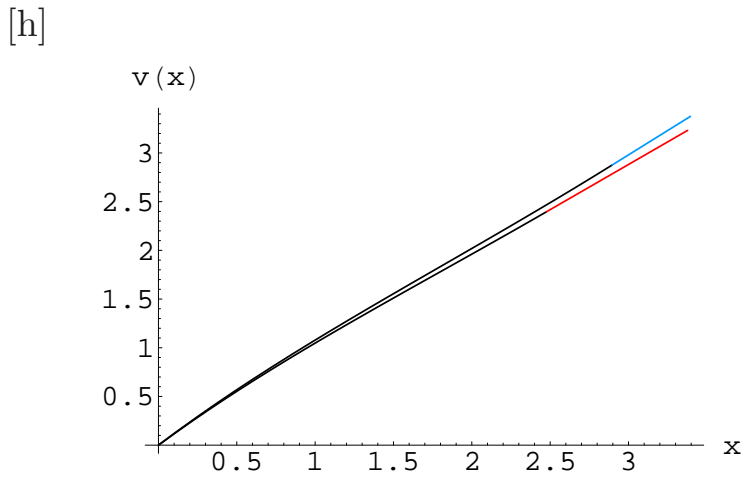

(b)

Figure 1. A numerical example of a Brownian motion with drift with parameters $(\mu, \alpha, \sigma, \lambda, \Delta)=$ $(0.3,0.15, \sqrt{2}, 0.1,0.25)$ : (a) The graph of $\beta(a)$ that attains the global maximum at $a^{*}=0.755$ with $\beta^{*}=1.443$. (b) The value function $v(x)$ (below) with $b^{*}=2.719$. It is compared with the case of $\Delta=0$ (above) with $\left(a_{0}, b_{0}, \beta_{0}\right)=(0.850,2.895,1.466)$.

\section{Conclusion}

We study optimal dividend payout problems with delay using various types of diffusions. Our method facilitates greatly the solution procedure due to the new characterization of the value function. The existence of the finite value function and the uniqueness of optimal threshold strategy reduce to verifications of the assumption of Proposition 4.1. Our models here are more realistic since the delays with respect to dividend payments are explicitly handled.

\section{REFERENCES}

[1] L. Alili, P. Patie, and J. L. Pedersen. Representations of the first hitting time density of an Ornstein-Uhlenbeck process. Stochastic Models, 21:967-980, 2005.

[2] L. H. R. Alvarez and J. Keppo. The impact of delivery lags on irreversible investment under uncertainty. European Journal of Operations Research, 136:173-180, 2002.

[3] A. Bar-Ilan and W. C. Strange. A model of sequential invetment. Journal of Economic Dynamics and Control, 22:437-463, 1998.

[4] E. Bayraktar and M. Egami. The effects of implementation delay on decision-making under uncertainty. Stochastic Processes and Their Applications, 117 (3):333-358, 2007.

[5] A. Bensoussan and J. L. Lions. Impulse Control and Quai-Variational Inequalities. Gauthier-Villars, Paris, 1982.

[6] A. N. Borodin and P. Salminen. Handbook of Brownian Motion Facts and Formulae. Birkhäuser, Boston, 2002.

[7] A. Cadenillas, S. Sarkar, and F. Zapatero. Optimal dividend policy with mean-reverting cash reservoir. Mathematical Finance, 17 (1):81-109.

[8] D. A. Darling and A. J. F. Siegert. The first passage problem for a continuous markov process. Annals of Mathematical Statistics, 24:624-639, 1953.

[9] S. Dayanik and I. Karatzas. On the optimal stopping problem for one-dimensional diffusions. Stochastic Processes and Their Applications, 107 (2):173-212, 2003. 
$[\mathrm{h}]$

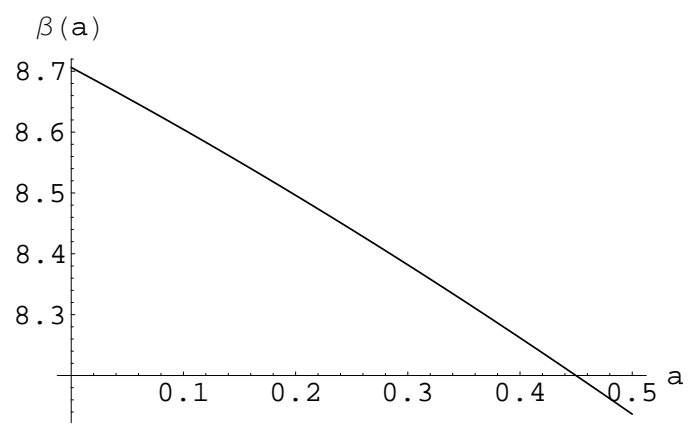

(a)

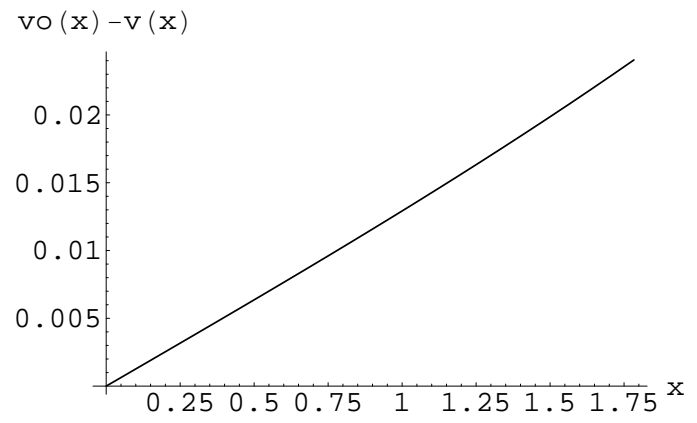

(c)

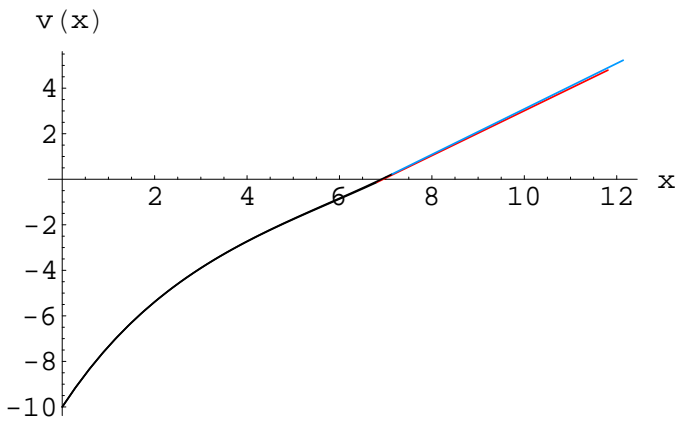

(e)

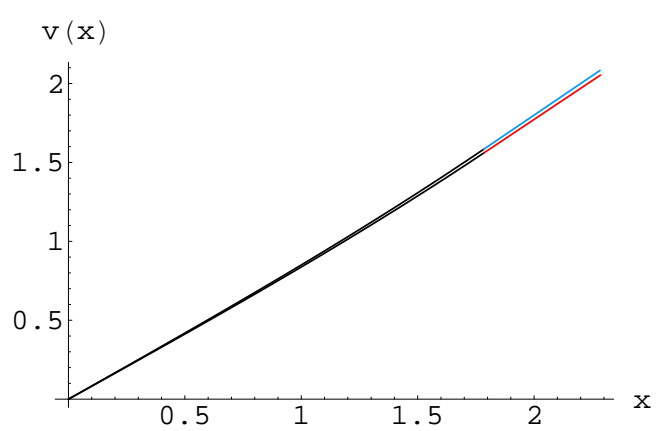

(b)

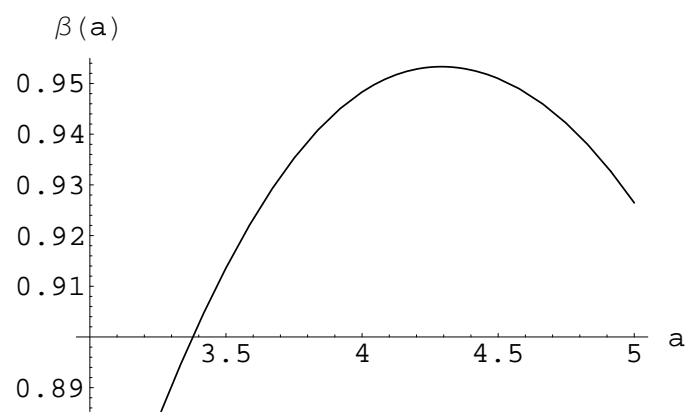

(d)

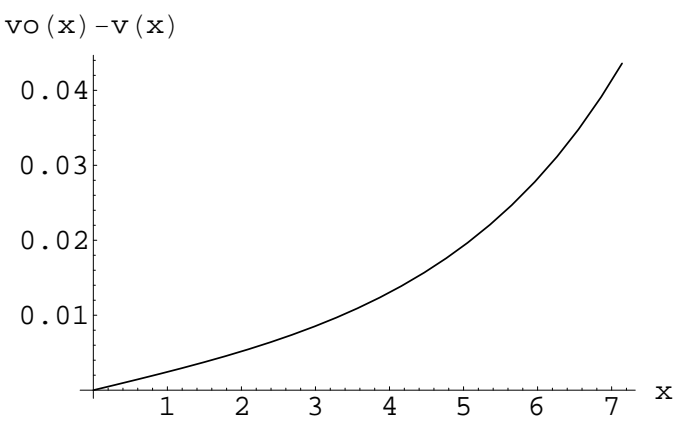

(f)

Figure 2. A numerical example of an OU process with parameters $(\rho, \alpha, \lambda, \Delta)=$ $(0.01,0.05,0.2,0.25)$ : (a) The graph of $\beta(a)$ that attains the global maximum at $a^{*}=0$ with $\beta^{*}=8.706$. (b) The value function $v(x)$ (below) with $b^{*}=1.785$. It is compared with the case $v_{0}(x)$ of $\Delta=0$ (above) with $\left(a_{0}, b_{0}, \beta_{0}\right)=(0,1.783,8.841)$. (c) Plot of the difference $v_{0}(x)-v(x)$. (d) In the case of penalty at ruin, $P=10$, the graph of $\beta(a)$ that attains the global maximum at $a^{*}=4.290$ with $\beta^{*}=0.953$. (b) The value function $v(x)$ (below) with $b^{*}=6.811$. It is compared with the case $v_{0}(x)$ of $\Delta=0$ (above) with $\left(a_{0}, b_{0}, \beta_{0}\right)=(4.349,7.141,0.979)$. (e) Plot of the difference $v_{0}(x)-v(x)$. 


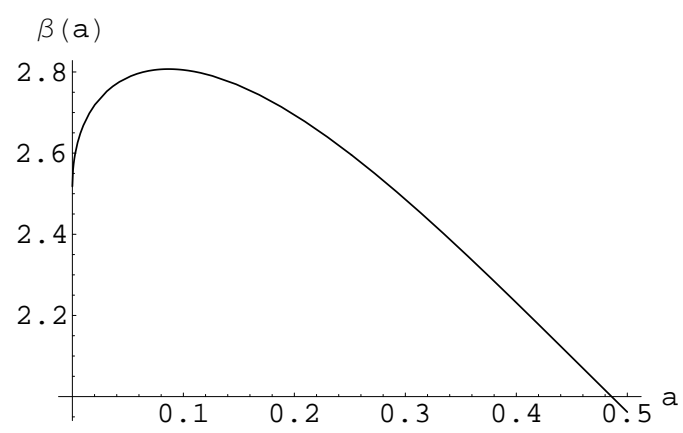

(a)

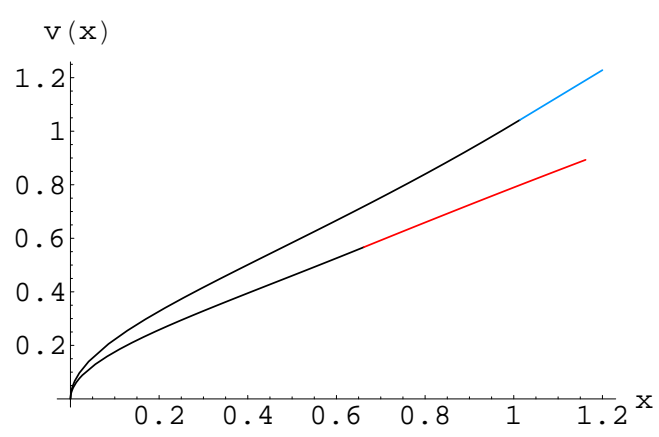

(b)

FiguRE 3. A numerical example of a square root process with parameters $(\rho, \alpha, \lambda, \Delta)=$ $(1,0.1,0.1,0.25)$ : (a) The graph of $\beta(a)$ that attains the global maximum at $a^{*}=0.09$ with $\beta^{*}=2.807$. ( b) The value function $v(x)$ (below) with $b^{*}=0.662$. It is compared with the case of $\Delta=0$ (above) with $\left(a_{0}, b_{0}, \beta_{0}\right)=(0.165,1.014,3.561)$.

[10] H. U. Gerber and E. S. W. Shiu. Geometric Brownian Motion Models for Assets and Liabilities : From Pension Funding to Optimal Dividends. North American Actuarial Journal, 7 (3):37-56, 2003.

[11] H. U. Gerber and E. S. W. Shiu. Optimal dividends: Analysis with Brownian motion. North American Actuarial Journal, 8 (1):1-20, 2004.

[12] H. U. Gerber, E. S. W. Shiu and H. Yang. Optimal Dividends in an Ornstein-Uhlenbeck Type Model with Credit and Debit Interest. North American Actuarial Journal, 10 (2):94-108, 2006.

[13] M. Jeanblanc-Picque and A. N. Shiryaev. Optimization of the flow of dividends. Russian Mat. Surveys, 50 (2):257-277, 1995.

[14] M. Jensen. Agency cost of free cash flow, corporate finance and takeovers. American Economic Reveiew, 76:323-329, 1986.

[15] I. Karatzas and S. E. Shreve. Brownian Motion and Stochastic Calculus. Springer-Verlag, New York, 1991.

[16] J. Keppo and S. Peura. Optimal bank capital with costly recapitalization. To appear in the Journal of Business, 2005.

[17] N. N. Lebedev. Special Functions and Their Applications. Dover Publications, New York, 1972.

[18] R. Merton. On the pricing of corporate debt: The risk structure of interest rates. Journal of Finance, 29:449-470, 1974.

[19] M. Musiela and M. Rutkowski. Martingale Methods in Financial Modelling. Springer, New York, 1997.

[20] B. Øksendal and A. Sulem. Applied Stochastic Control of Jump Diffusions. Springer Verlag, New York, 2005.

[21] B. Øksendal and A. Sulem. Optimal stochastic impulse control with delayed reaction. Preprint. University of Oslo, 2005.

[22] J. Paulsen. Optimal dividend payouts for diffusions with solvency constraints. Finance and Stochastics, 7:457-473, 2003.

[23] J. Paulsen. Optimal dividend payments until ruin of diffusion processes when payments are subject to both fixed and proportional costs. Advances in Applied Probability, 3:669-689, 2007.

[24] M. Rubinstein and E. Reiner. Breaking down the barriers. Risk, September:28-35, 1991.

[25] A. Subramanian and R. A. Jarrow. The liquidity discount. Mathematical Finance, 11:447-474, 2001.

[26] M. Taksar. Optimal risk and dividend distribution control models for an insurance company. Mathematical Methods of Operations Research, 51:1-42, 2000. 
$[\mathrm{h}]$

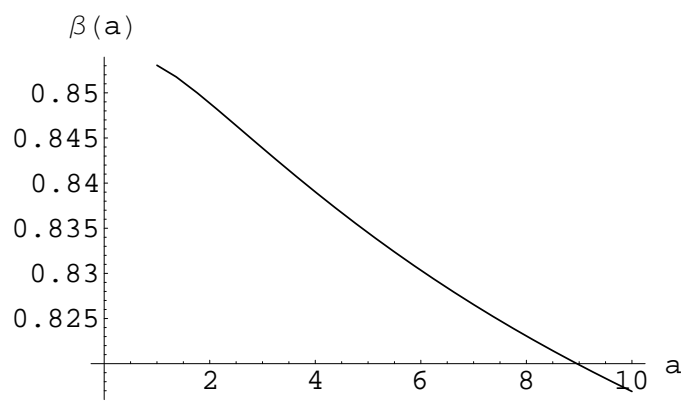

(a)

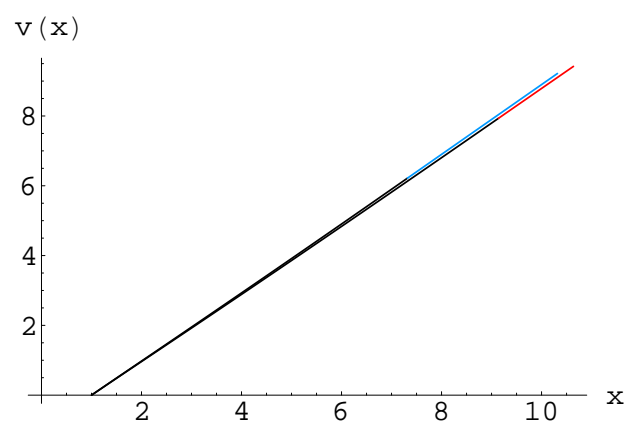

(b)

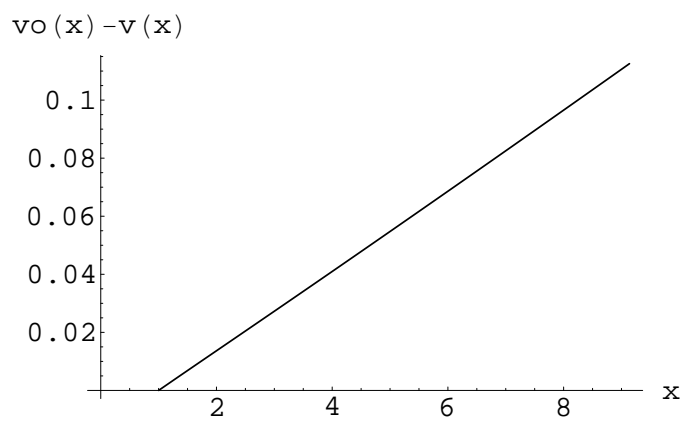

(c)

FiguRE 4. A numerical example of an geometric Brownian motion with parameters $(\mu, \sigma, \alpha, \lambda, \Delta)=(0.05, \sqrt{2}, 0.1,0.1,0.25)$ and the ruin level $d=1$ : (a) The graph of $\beta(a)$ that attains the global maximum at $a^{*}=1$ with $\beta^{*}=0.853$. (b) The value function $v(x)$ (below) with $b^{*}=9.138$. It is compared with the case of $\Delta=0$ (above) with $\left(a_{0}, b_{0}, \beta_{0}\right)=(1,7.318,0.865)$. (c) Plot of the difference $v_{0}(x)-v(x)$.

(E. Bayraktar) Department of Mathematics, University of Michigan, Ann Arbor, Mi 48109

E-mail address: erhan@umich.edu

(M. Egami) Graduate School of Economics, Kyoto University, Sakyo-Ku, Kyoto, 606-8501, Japan E-mail address: egami@econ.kyoto-u.ac.jp 